\title{
An Optimization of Granular Networks Based on PSO and Two-Sided Gaussian Contexts
}

\author{
Keun-Chang Kwak \\ Dept. of Control, Instrumentation, and Robot Engineering \\ Chosun University, 375 Seosuk-Dong \\ Gwangju, Korea
}

\begin{abstract}
This paper is concerned with an optimization of GN (Granular Networks) based on PSO (Particle Swarm Optimization) and Information granulation). The GN is designed by the linguistic model using context-based fuzzy c-means clustering algorithm performing relationship between fuzzy sets defined in the input and output space. The contexts used in this paper are based on two-sided Gaussian membership functions. The main goal of optimization based on PSO is to find the number of clusters obtained in each context and weighting factor. Finally, we apply to coagulant dosing process in a water purification plant to evaluate the predication performance and compare the proposed approach with other previous methods.
\end{abstract}

Keywords-granular networks; particle swarm optimization; linguistic model; two-sided Gaussian contexts.

\section{INTRODUCTION}

Granular computing is a general computation theory for effectively using granules such as classes, clusters, subsets, groups and intervals to build an efficient computational model for complex applications with huge amounts of data, information and knowledge. Though the label is relatively recent, the basic notions and principles of granular computing, though under different names, have appeared in many related fields, such as information hiding in programming, granularity in artificial intelligence, divide and conquer in theoretical computer science, interval computing, cluster analysis, fuzzy and rough set theories, quotient space theory, belief functions, machine learning, databases, and many others. Furthermore, granular computing forms a unified conceptual and computing platform [1]. Yet, it directly benefits to form the already existing and well-established concepts of information granules formed in set theory, fuzzy sets, rough sets and others. In order to form notional and calculative platform of granular computing in conjunction with linguistic model using fuzzy clustering directly, we develop a design methodology of granular networks. This network indicates a relationship among fuzzy congregating forming from input and output space and expressing information granules. The linguistic context forming this relationship is admitted by a developer of the system, and information granules are constructed by using context-based fuzzy c-means) clustering. However, this network is difficult to find the number of clusters generated by each context and weighting factor related to fuzzy clustering [2-5]. Therefore, we perform the optimization of granular networks using particle swarm optimization which is one of evolutionary computation methods respectively and compare these performances. Particle swarm optimization is based on social behavior of bird flocking or fish schooling. This method has features that use parallel processing and an objective function for solving problem [6-10]. In the design of granular networks, these contexts were generated through a series of triangular membership functions with equally spaced along the domain of an output variable. However, we may encounter a data scarcity problem due to small data included in some linguistic context [11][12]. Thus, this problem brings about the difficulty to obtain fuzzy rules from the context-based fuzzy cmeans clustering. Therefore, we use a probabilistic distribution of output variable to produce the flexible linguistic contexts from two-sided Gaussian type-based membership function[13]. Finally, we demonstrate the superiority and effectiveness of predication performance for coagulant dosing process in a water purification plant [14][15].

\section{GRANULAR NETWORKS}

In this section, we describe the concept of granular networks based on linguistic model and information granulation. The granular networks belong to a category of fuzzy modeling using directly basic idea of fuzzy clustering. This clustering technique builds information granules in the form of fuzzy sets and develops clusters by preserving the homogeneity of the clustered patterns associated with the input and output space. The numerical formula of this membership matrix $U$ of clustering is computed as follows

$$
u_{i k}=f_{k} / \sum_{j=1}^{c}\left(\frac{\left\|\mathrm{x}_{k}-\mathrm{c}_{i}\right\|}{\left\|\mathrm{x}_{k}-\mathrm{c}_{j}\right\|}\right)^{2 /(m-1)}
$$

where $m \in[1, \infty]$ is a weighting factor. Here the $f_{k}$ is obtained by the membership degree between 0 and 1 . The $f_{k}=\mathrm{T}\left(d_{\mathrm{k}}\right)$ represents a level of involvement of the $k$ 'th data in the assumed contexts of the output space. Fuzzy set in output space is defined by $T: D \rightarrow[0,1]$. This is a universe of discourse of output. For this reason, we modify the requirements of the membership matrix as follows

$$
\mathrm{U}(\mathrm{f})=\left\{u_{i k} \in[0,1] \mid \sum_{i=1}^{c} u_{i k}=f_{k} \forall k \text { and } 0<\sum_{k=1}^{N} u_{i k}<N \forall i\right\}
$$

The linguistic contexts to obtain $f_{k}$ are generated through a series of trapezoidal membership functions along the domain of 
an output variable and a 1/2 overlap between successive fuzzy sets as shown in Fig. 1 [2]. However, we may encounter a data scarcity problem due to small data included in some linguistic context. Thus, this problem brings about the difficulty to obtain fuzzy rules from the context-based fuzzy c-means clustering. Therefore, we use a probabilistic distribution of output variable to produce the flexible linguistic contexts. Fig. 2 shows the automatic generation of linguistic contexts with triangular membership function [13]. Finally, we change triangular contexts into two-sided Gaussian contexts to deal with nonlinearity characteristics to be modeled. The two-sided Gaussian contexts shown in Fig. 3 are a combination of two of Gaussian membership functions. The left membership function, specified by first sig1(sigma) and c1(center), determines the shape of the leftmost curve. The right membership function determines the shape of the rightmost curve. Whenever $\mathrm{c} 1<\mathrm{c} 2$, the two-sided Gaussian contexts reach a maximum value of 1 . Otherwise, the maximum value is less than one.

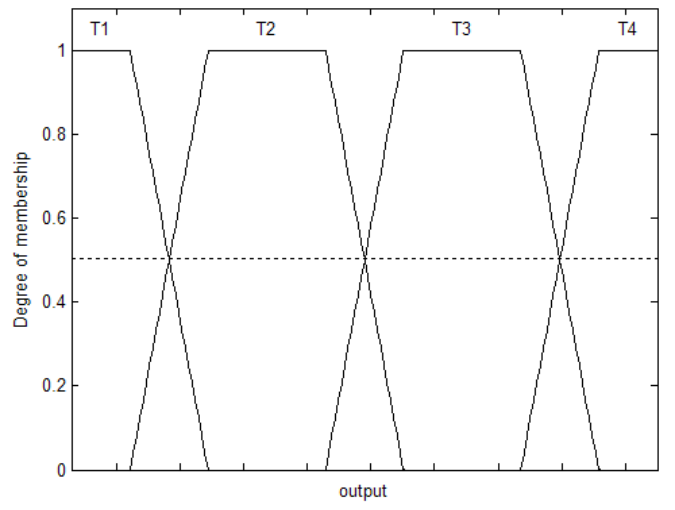

Figure 1. Conventional trapezoidal contexts

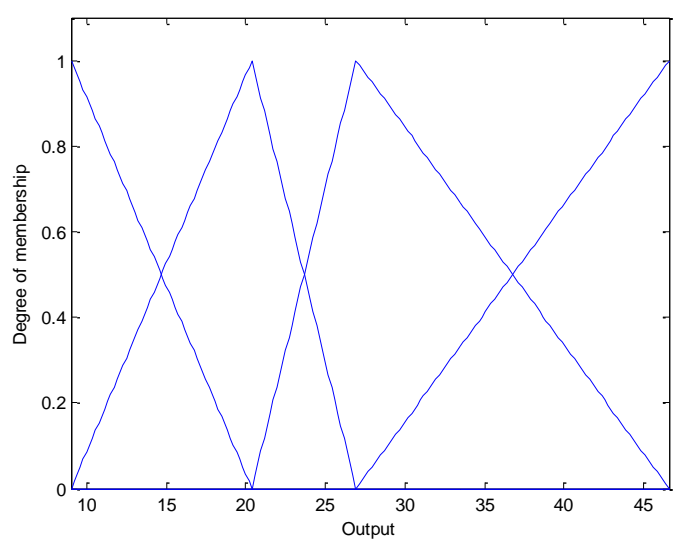

Figure 2. Flexible triangular contexts

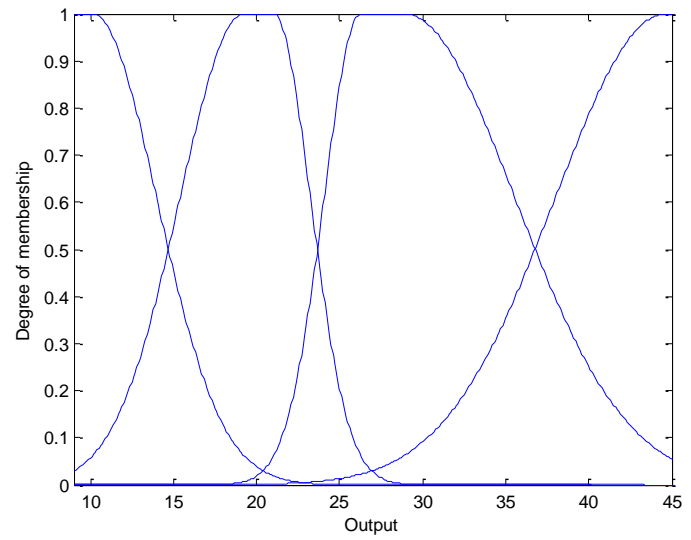

Figure 3. Flexible two-sided Gaussian contexts

The center of clusters generated from each context is expressed as follows

$$
u_{i}=\sum_{k=1}^{N} u_{i k}^{m} x_{k} / \sum_{k=1}^{N} u_{i k}^{m}
$$

Fig. 4 shows the architecture of granular networks with four layers. The premise parameter of the first layer consists of the cluster centers obtained through context-based fuzzy c-means clustering. The consequent parameter is composed of linguistic contexts produced in output space. The network output $\mathrm{Y}$ with interval value is computed by fuzzy number as follows

$$
Y=\sum_{\oplus} W_{t} \otimes z_{t}
$$

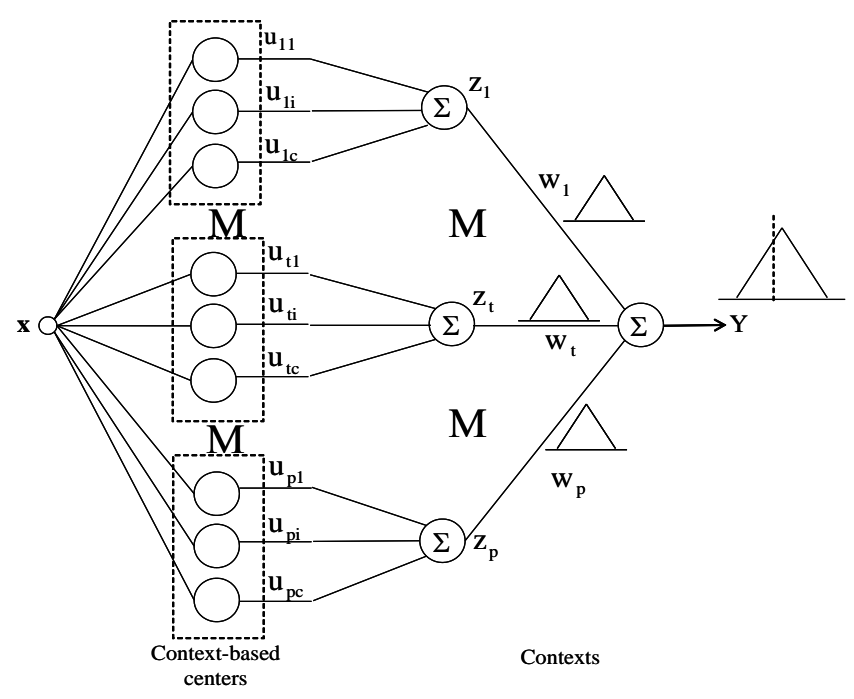

Figure 4. Architecture of granular networks 
Fig. 5 visualizes the cluster centers generated by each context. Here square box represents cluster centers. The number of cluster centers in each context is 4 . The four if-then rules are produced within the range of each context. Fig. 6 shows 16 evident clusters generated by the context-free fuzzy clustering algorithm (FCM clustering). However, these clusters change when we reflect the corresponding output value. In contrast to Fig. 6, Fig. 5 shows clusters to preserve homogeneity with respect to the output variable. We can recognize from Fig. 5 that the clusters obtained from contextbased fuzzy clustering algorithm have the more homogeneity than those produced by context-free fuzzy clustering.
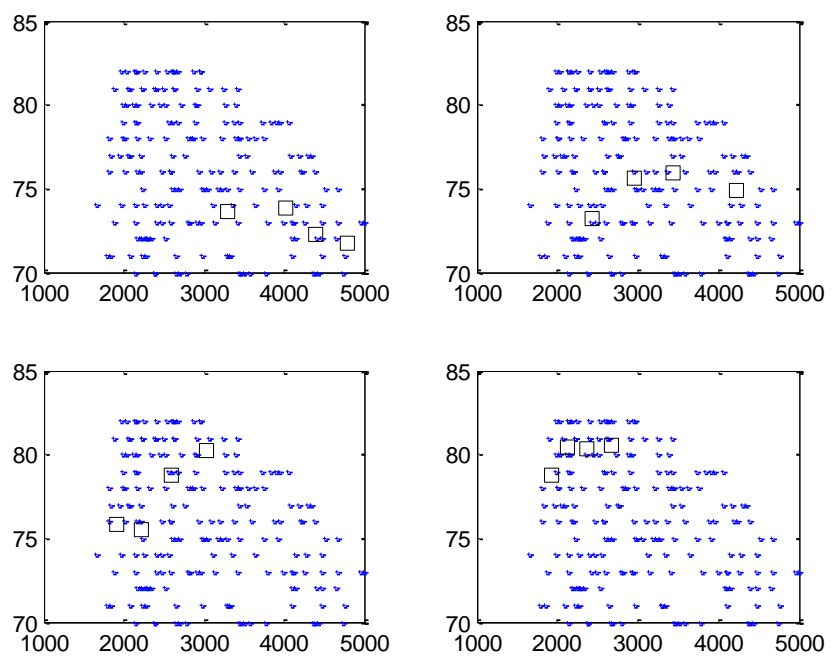

Figure 5. Cluster centers generated by each context $(\mathrm{CFCM}, \mathrm{p}=\mathrm{c}=4)$

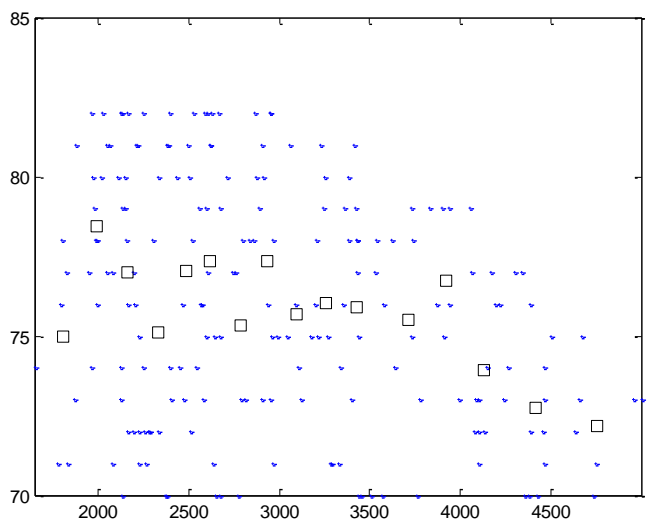

Figure 6. Cluster centers generated by each context (FCM, c=16)

\section{PARTICLE SWARM OPTIMIZATION}

The PSO method is one of swarm intelligence methods for solving the optimization problems. The PSO algorithm proposed by Kennedy is performed by social behavior of bird flocking or fish schooling. The character of PSO easily can handle fitness function for solving complex problems. Furthermore, it can control a relationship between global and local search.
Here, each particle adjusts information of location with experience of them and their neighborhood. It can form the answer of optimum in short time.

As the velocity of particle movement of PSO is only demanded, it is easy to be embodiment and brevity of a theory. The basic element of PSO is simply as follows

Particle: individual belonged swarm.

Swarm: a set of particles.

Pbest: particle had located information of optimum. Pbest.

Gbest: particle had located information of optimum in

Velocity: velocity of movement in particles.

The velocity is computed as follows

$$
\begin{aligned}
v_{j k}(t+1)= & w(t) \cdot v_{j k}(t) \\
& +c_{1} \cdot r_{1} \cdot\left(\text { pbest }_{j k}(t)-x_{j k}(t)\right) \\
& +c_{2} \cdot r_{2} \cdot\left(\text { gbest }_{k}(t)-x_{j k}(t)\right)
\end{aligned}
$$

where $x_{j k}(t)$ is position of dimension $k$ of particle $j$ at time $t$. $w$ is an inertia weight factor. $v_{j k}(t)$ is a velocity of particle $j$ at time $t . c_{1}$ and $c_{2}$ are cognitive and social acceleration factors respectively. $r_{1}$ and $r_{2}$ are random numbers uniformly distributed in the range $(0,1)$, pbest $_{j k}(t)$ is best position obtained by particle $j$. gbest $t_{k}(t)$ is best position obtained by the whole swarm. The optimization stage using PSO algorithm is as follows

[Step 1] Set the initial parameters of PSO: the size of swarms, the number of max iteration, a dimension, recognition, sociality, the range of velocity of movement $\left[-v_{k \max }, v_{k \max }\right]$, the range of cluster, the range of weighting factor.

[Step 2] Compute the output values of granular networks

[Step 3] Compute the fitness function from each particle. Here, we use RMSE (root mean square error) between the network output and actual output on training data and test data. Here $\theta$ is the adjustment factor. We set to 0.5 .

$$
F=\frac{1}{Q_{\text {trnRMSE }} \bullet \theta+Q_{\text {chkRMSE }} \bullet(1-\theta)}
$$

[Step 4] Adjust scaling by $F=F-\min (F)$ to maintain the positive values.

[Step 5] Compute the localization information of particle as follows

$$
x_{j k}(t)=v_{j k}(t)+x_{j k}(t-1)
$$

[Step 6] If it satisfied with condition of a conclusion, stop the search process, otherwise go to the [Step 3]. 


\section{CONCLUSIONS}

In this section, we shall apply to coagulant dosing process in water purification plant to evaluate the predication performance. Also, we shall compare the proposed approach with other previous methods. The field test data of this process to be modeled is obtained at the Amsa water purification plant, Seoul, Korea, having a water purification capacity of $1,320,000$ ton/day. We use the successive 346 samples among jar-test data for one year. The input consists of four variables, including the turbidity of raw water, temperature, $\mathrm{pH}$, and alkalinity. The output variable is Poli-Aluminum Chloride widely used as a coagulant. In order to evaluate the resultant model, we divide the data sets into training and checking data sets. Here we choose 173 training sets for model construction, while the remaining data sets are used for model validation. Firstly we confine the search domain such as the number of clusters from 2 to 9 in each context and weighting factor from 1.5 to 3 , respectively. Here we set to $p=8$. Furthermore, we used 8 bit binary coding for each variable. Each swarm contains 100 particles. Also, we linearly used inertia weight factor from 0.9 to 0.4 .

Fig. 7 visualizes the two-sided Gaussian contexts when $\mathrm{p}=8$. As shown in Fig. 7, we encountered a data scarcity problem due to small data included in some context (eighth context). Thus, this problem can be solved by using flexible Gaussian contexts obtained from probabilistic distribution. Fig. 8 shows the predication performance for checking data set. As shown in Fig. 8, the experimental results revealed that the proposed method showed a good predication performance. Table 1 lists the comparison results of predication performance for training and checking data set, respectively. As listed in Table 1, the proposed method outperformed the LR(Linear Regression, neural networks by (MLP) Multilayer Perceptron, and RBFN (Radial Basis Function Network) based on CFCM (Context-based Fuzzy c-means Clustering).

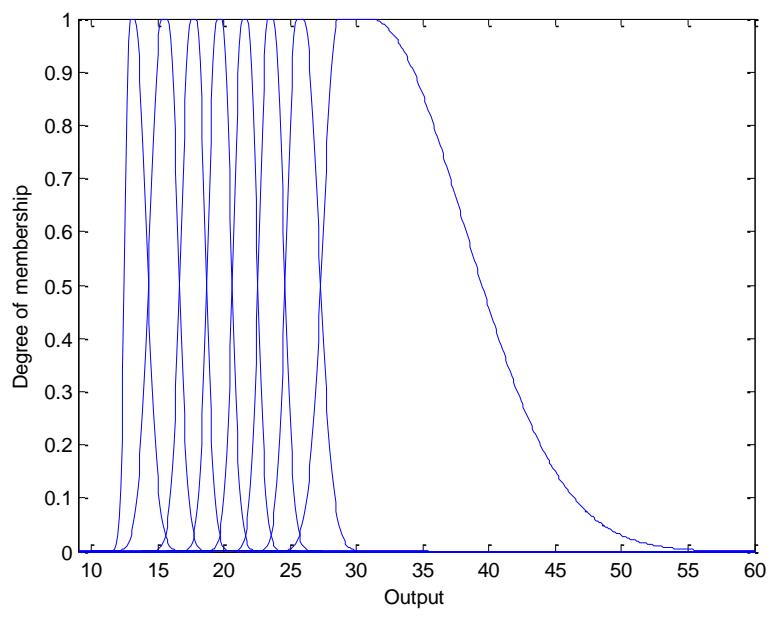

Figure 7. Two-sided Gaussian contexts $(\mathrm{p}=8)$

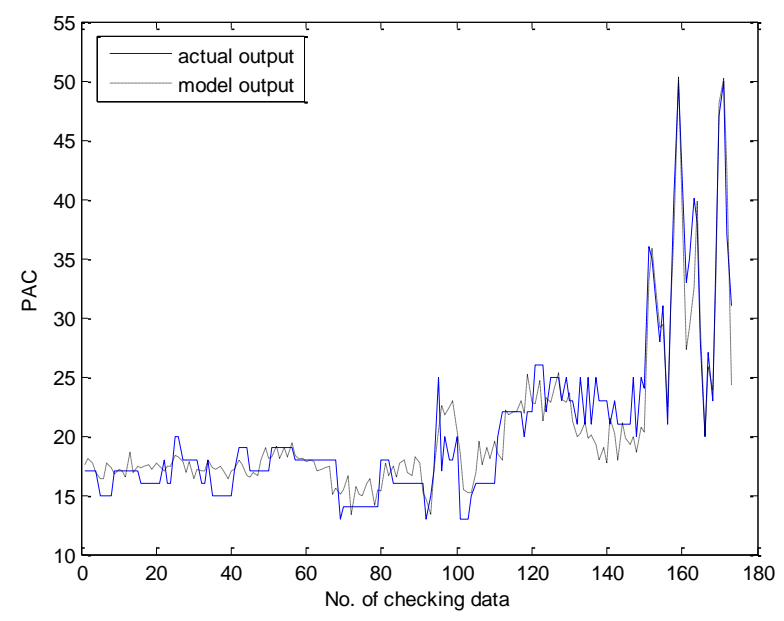

Figure 8. Prediction performance for checking data

TABLE I. COMPARISON RESULTS

\begin{tabular}{|c|c|c|}
\hline & $\begin{array}{c}\text { RMSE } \\
\text { (Training data) }\end{array}$ & $\begin{array}{c}\text { RMSE } \\
\text { (Checking data ) }\end{array}$ \\
\hline \hline LR & 3.508 & 3.578 \\
\hline MLP & 3.191 & 3.251 \\
\hline LMFN-CFCM [11] $[2]$ & 3.048 & 3.219 \\
\hline LR-QANFN [14] & 1.939 & 3.788 \\
\hline $\begin{array}{c}\text { The proposed method } \\
\text { (PSO-GN) }\end{array}$ & 1.661 & 2.196 \\
\hline
\end{tabular}

\section{CONCLUSIONS}

We developed the PSO-based granular networks based on information granulation. Furthermore, we used flexible twosided Gaussian contexts produced from output domain to deal with non-linearity characteristics to be modeled. We demonstrated the effectiveness through the experimental results of prediction performance in comparison to the previous works. Finally, we formed notional and calculative platform of granular computing in conjunction with granular networks using context-based fuzzy clustering. Granular computing is expected to come new market challenge to software companies. It is expected to be a core technique of IT convergence, ubiquitous computing environments, and intelligent knowledge research that supports knowledge-based society.

\section{REFERENCES}

[1] W. Pedrycz, A. Skowron, and V. Kreinovich, Handbook of Granular Computing, John Wiley \& Sons, 2008.

[2] W. Pedrycz and A. V. Vasilakos, "Linguistic models and linguistic modeling", IEEE Trans. on Systems, Man, and Cybernetics-Part C, Vol.29, No.6, 1999, pp. 745-757. 
[3] W. Pedrycz and K. C. Kwak, "Linguistic models as framework of usercentric system modeling", IEEE Trans. on Systems, Man, and Cybernetics-Part A, Vol.36, No.4, 2006, pp.727-745.

[4] W. Pedrycz, "Conditional fuzzy c-means", Pattern Recognition Letters, Vol.17, 1996, pp.625-632.

[5] W. Pedrycz and K. C. Kwak, "The development of incremental models", IEEE Trans. on Fuzzy Systems, Vol.15, No.3, 2007, pp.507-518.

[6] J. Kennedy and R. Eberhart, "Particle swarm optimization", IEEE Int. Conf. Neural Networks, Vol. IV, 1995, pp.1942-1948.

[7] M. A. Abido, "Optimal design of power system stabilizers using particle swarm optimization", IEEE Trans, Energy Conversion, Vol.17, No.3, 2002, pp.406-413.

[8] K. F. Parsopoulos, "On the computation of all global minimizes through particle swarm optimization", IEEE Trans. Evolutionary Computation, Vol.8, No.3, 2004, pp.211-224.

[9] J. Kennedy, "The particle swarm: Social adaptation of knowledge", IEEE Int. Conf. Evolutionary Computation, 1997, pp.303-308.

[10] S. Panda, N. P. Padhy, "Comparison of particle swarm optimization and genetic algorithm for TCSC-based controller design", International Journal of Computer Science and Engineering, Vol.1, No.1, 2007, pp.4149.

[11] W. Pedrycz, "Conditional fuzzy clustering in the design of radial basis function neural networks", IEEE Tans. on Neural Networks, Vol.9, No.4, 1999, pp.745-757.

[12] S. S. Kim, H. J. Choi. K. C. Kwak "Knowledge extraction and representation using quantum mechanics and intelligent models", Expert Systems with Applications, Vol. 39, pp. 3572-3581, 2012.

[13] G. Panoutsos, M. Mahfouf, G. H. Mills, B. H. Brown, "A generic framework for enhancing the interpretability of granular computingbased information", 5th IEEE International Conference Intelligent Systems, London, UK, 2010, pp. 19-24.

[14] S. S. Kim, K. C. Kwak, "Development of Quantum-based Adaptive Neuro-Fuzzy Networks", IEEE Trans. on Systems, Man, and Cybernetics-Part B, Vol. 40, No. 1, pp. 91-100, 2010.

[15] Y. H. Han, K. C. Kwak , "An optimization of granular network by evolutionary methods", AIKED10, Univ. of Cambridge, UK, 2010, pp.65-70.

\section{AUTHOR PROFILE}

Keun-Chang Kwak received the B.Sc., M.Sc., and Ph.D. degrees from Chungbuk National University, Cheongju, Korea, in 1996, 1998, and 2002, respectively. During 2003-2005, he was a Postdoctoral Fellow with the Department of Electrical and Computer Engineering, University of Alberta, Edmonton, AB, Canada. From 2005 to 2007, he was a Senior Researcher with the Human-Robot Interaction Team, Intelligent Robot Division, Electronics and Telecommunications Research Institute, Daejeon, Korea. He is currently the Assistant Professor with the Department of Control, Instrumentation, and Robot Engineering, Chosun University, Gwangju, Korea. His research interests include human-robot interaction, computational intelligence, biometrics, and pattern recognition. Dr. Kwak is a member of IEEE, IEICE, KFIS, KRS, ICROS, KIPS, and IEEK. 